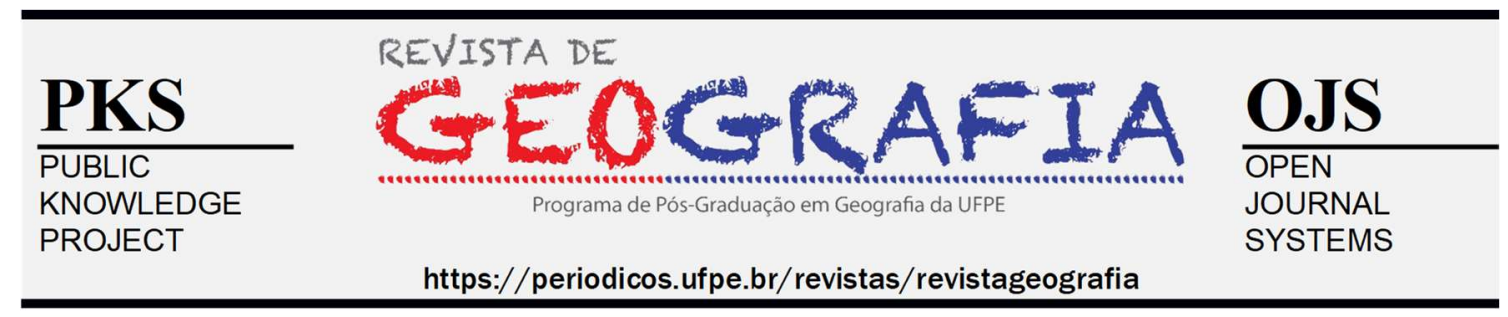

\title{
CARACTERIZAÇÃO MORFOMÉTRICA DAS BACIAS DE CONTRIBUIÇÃO DOS RESERVATÓRIOS UMARI E MENDUBIM, SEMIÁRIDO DO RIO GRANDE DO NORTE, BRASIL
}

\author{
Herbter Ranielle Lira De-Carvalho ${ }^{1}$, Gustavo Herique Gonzaga da Silva Henry-Silva²
}

${ }^{1}$ Universidade Federal Rural do Semiárido (UFERSA), Instituto Brasileiro do Meio Ambiente e Recursos Naturais Renováveis (IBAMA), Analista Ambiental, Geoprocessamento. E-mail: prof.herbster.rlcarvalho@gmail.com; http://orcid.org/0000-0002-2446-7065

2 Coordenador do Laboratório de Limnologia e Qualidade de Água do Semiárido (LIMNOAQUA), Universidade Federal Rural do Semiárido (UFERSA). E-mail: gustavo@ufersa.edu.br; http://orcid.org/0000-0001-5067-225X

Artigo recebido em 09/06/2020 e aceito em 17/11/2020

\begin{abstract}
RESUMO:
Há carência de dados morfométricos de sub-bacias hidrográficas do Nordeste brasileiro. Em geral, os trabalhos são direcionados para a caracterização de grandes bacias, sendo raramente encontrados trabalhos voltados para as bacias de contribuição de reservatórios. Neste contexto, o presente trabalho teve como objetivo caracterizar as bacias de contribuição de dois reservatórios no semiárido do Rio Grande do Norte, Umari e Mendubim, aplicando técnicas de geoprocessamento em modelos digitais de elevação (MDE), gerados e disponibilizados, de forma gratuita, pelo Instituto Nacional de Pesquisa Espaciais (INPE), a partir de imagens do programa "Shuttle Radar Topography Mission" (SRTM). O processamento dos MDEs foi realizado utilizando o programa livre QGIS 2.18. A partir dos dados extraídos foram realizadas as análises hipsométricas e morfométricas das bacias em estudo. Ambas as bacias possuem formatos, predominantemente, alongados, com pequena tendência a enchentes, especialmente a bacia de contribuição do reservatório de Umari. As redes de drenagem possuem baixa densidade, declividade e velocidade de escoamento, com substratos permeáveis e com relativo equilíbrio entre escoamento e infiltração. $O$ geoprocessamento de MDEs e a aplicação de ferramentas disponíveis no QGis 2.18 se mostraram eficientes na obtenção das informações necessárias ao gerenciamento de reservatórios e de suas bacias de contribuição.
\end{abstract}

Palavras-chave: QGis; geoprocessamento; hipsometria; parâmetros morfométricos; sub-bacias hidrográficas

\section{MORPHOMETRIC CHARACTERIZATION OF THE CONTRIBUTION BASINS OF THE UMARI AND MENDUBIM RESERVOIRS, SEMIARID OF RIO GRANDE DO NORTE, BRAZIL}

\begin{abstract}
:
There is a lack of morphometric data for sub-basins in Northeast Brazil. In general, the works are directed to the characterization of large basins, with rarely being found works focused on the reservoir contribution basins. In this sense, the present work aimed to characterize the contribution basins of two reservoirs in the semiarid region of Rio Grande do Norte, Umari, and Mendubim, applying geoprocessing techniques in digital elevation models (MDE), generated and made available, free of charge, by the National Institute of Space Research (INPE), from images of the program "Shuttle Radar Topography Mission" (SRTM). The processing of the MDEs was performed using the free program QGIS 2.18. From the extracted data,
\end{abstract}


hypsometric and morphometric analyses of the basins under study were performed. Both basins have predominantly elongated shapes, with little tendency to flood, especially the contribution basin of the Umari reservoir. The drainage networks have low density, slope and flow speed, with permeable substrates and with a relative balance between flow and infiltration. The geoprocessing of MDEs and the application of tools available in QGis 2.18 proved to be efficient in obtaining the necessary information for the management of reservoirs and their contribution basins.

Keywords: QGis; geoprocessing; hypsometry; morphometric parameters; Umari; Mendubim

\section{INTRODUÇÃO}

A gestão dos recursos hídricos, principalmente em regiões do semiárido, vem sendo uma das grandes preocupações, tanto das populações quanto das autoridades responsáveis pelo uso adequado desses recursos. A Política Nacional de Recursos Hídricos, que criou o Sistema Nacional de Gerenciamento dos Recursos Hídricos (BRASIL, 1997), considera que a água é um bem de domínio público, um recurso limitado e de valor econômico, instituindo as bacias hidrográficas como unidades territoriais, cuja gestão deve ser descentralizada e partilhada entre o Poder Público, usuários e comunidades. Neste contexto, é importante a caracterização morfométrica das bacias como um dos instrumentos de gestão e análise da potencialidade hídrica de uma região, permitindo avaliar e propor ações que visem à utilização sustentável dos recursos hídricos, com a elaboração e execução de estratégias de gestão (AHER; ADINARAYANA; GORANTIWAR, 2014; MENEZES et al., 2014).

Atualmente, o uso de imagens de satélites na análise e gerenciamento de bacias hidrográficas é cada vez maior (YUSUF; MURTILAKSONO et al., 2016). O estudo de parâmetros como relevo, tipos de solo, seu uso e ocupação, flora e suas relações, são de suma importância na descrição e análise da dinâmica de bacias hidrográficas (MEDEIROS et al., 2019). Como é o caso dos mapas auto organizados que foram utilizados para caracterizar a hidrogeologia do aquífero Serra-Geral no estado do Paraná; ou dos sistemas de informações geográficas e "Revised Universal Soil Loss Equation" (RUSLE), que se mostraram eficientes na modelagem da erosão na bacia hidrográfica do córrego Indaiá, na planície pantaneira do Mato Grosso do Sul (CUNHA; BACANI; PANACHUKI, 2016; IWASHITA, FRIEDEL e FERREIRA, 2017).

Técnicas de geoprocessamento, aplicadas a Modelos digitais de elevação (MDEs), oriundos de dados da "Shuttle Radar Topography Mission" (SRTM), revelaramse importantes na análise da relação entre a morfologia da rede de drenagem, das formas de relevo e da cobertura do solo na bacia do baixo médio São Francisco (SILVA et al., 
2014). Dados SRTM foram usados com sucessos na seleção de áreas de várzeas da bacia do rio Paraíba do Sul a serem submetidas à renaturalização das funções hidrológicas (BAPTISTA et al., 2014), mostrando-se como uma ferramenta eficiente na gestão de bacias hidrográficas e de seus corpos hídricos. No entanto, no Nordeste brasileiro existe uma lacuna na caracterização morfométrica de sub-bacias, com ênfase para as de contribuição de reservatórios, sendo que a maioria dos trabalhos de análise morfométrica encontrados na literatura estão voltados para grandes bacias hidrográficas.

Neste contexto, objetivamos com o presente estudo caracterizar os parâmetros morfométricos e altimétricos das bacias de contribuição de dois reservatórios do semiárido potiguar: Umari e Mendubim, utilizando técnicas de geoprocessamento e imagens do projeto TOPODATA.

\section{MATERIAL E MÉTODOS}

Área de estudo

Os reservatórios de Umari e Mendubim estão entre os dez maiores açudes do estado do Rio Grande do Norte. O reservatório de Umari (543'22,57"S; 37²14'48,35" W) está localizado no município Upanema, a $8 \mathrm{~km}$ da sede municipal, e é administrado pela Companhia de Água e Esgoto do Estado do Rio Grande do Norte (CAERN). Este reservatório é resultante do barramento do rio do Carmo, sendo a terceira maior barragem potiguar. A bacia de contribuição do reservatório Umari é uma das sub-bacias da bacia hidrográfica do rio Apodi-Mossoró, que corresponde a 26,8\% do território do Rio Grande do Norte (SOUZA; SILVA; DIAS, 2012). Resultante do barramento do rio Paraú, o reservatório Mendubim (05²0'6,62" S; 3656'59.75" W), distante $15 \mathrm{~km}$ da sede municipal de $A c ̧ u / R N$, tem sua bacia de contribuição inserida na bacia hidrográfica do rio Piancó-Piranhas-Açu que, por sua vez, é uma bacia federal (OLIVEIRA; BARBOSA; DANTAS NETO, 2013) (Figura 1).

De acordo com a classificação citada por Bentes-Gama (2003), as bacias hidrográficas desses reservatórios são caracterizadas como exorreicas, deficitárias e intermitentes. Em função da classificação de Köppen e Geiger (1936), as bacias possuem clima do tipo BSw'h', clima quente e semiárido, com estações chuvosas mal distribuídas e com atraso para o outono. Quanto ao solo, a bacia de contribuição do reservatório Umari tem a maior parte de sua área formada por solo Argissolo vermelho-amarelo de textura arenosa, com baixa retenção de nutrientes, profundidade variando de média a profunda e 
topografia plana e baixa. A bacia do reservatório Mendubim e formada predominantemente por solo Bruno não Cálcico e litossolo, característicos da porção Figura 1: Localização e hidrografia das bacias de contribuição dos reservatórios Umari e Mendubim, Região Nordeste (NE) do Brasil.

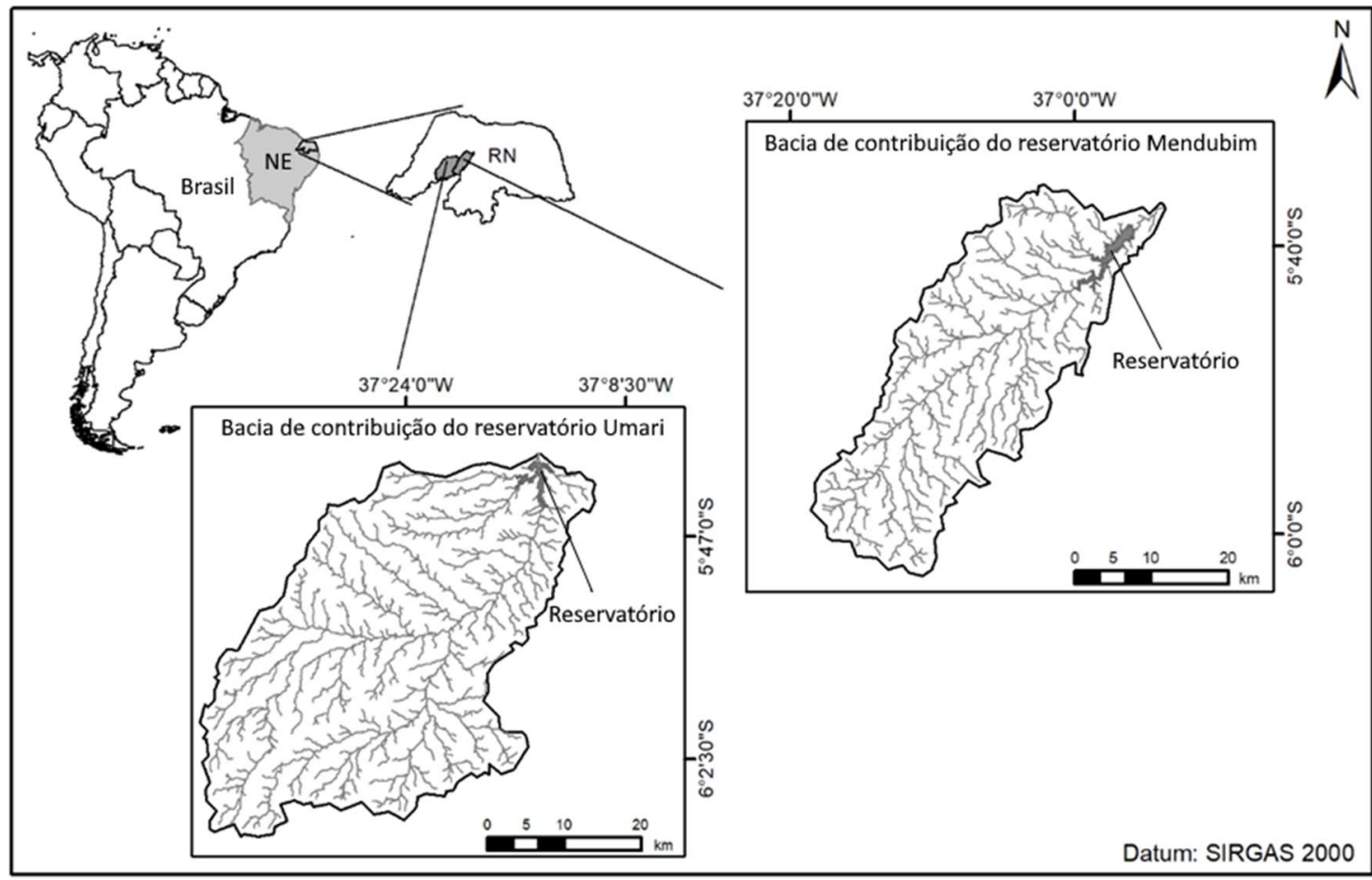

potiguar da bacia hidrográfica Piancó-Piranhas-Açu (RÊGO; TRAJANO, 2005).

Fontes: IBGE 2010, INPE 2009, elaborado pelos autores a partir do geoprocessamento de modelos digitais de elevação (MDEs) dispoinibilizados pelo Projeto TOPODATA (INPE, 2009).

Aquisição e processamento de imagens TOPODATA

No presente trabalho utilizamos Modelos Digitais de Elevação (MDEs) elaborados pelo Projeto TOPODATA do Instituto Nacional de Pesquisas Espaciais (INPE), a partir de imagens oriundas da missão do projeto internacional da Agência Americana de Inteligência Geoespacial (NGA) conhecida como "Shutlle Radar Topography Mission” (SRTM), e disponibilizadas pelo “United States Geological Survey (USGS)". O uso desse tipo de imagens é bem diversificado, dada à qualidade e precisão das mesmas. O projeto SRTM teve como objetivo fazer o levantamento das áreas continentais através da elaboração de modelos digitais de elevação (MDEs). Foram usadas técnicas de interferometria, utilizando radares nas bandas $\mathrm{C}$ e X, entre os paralelos $56^{\circ} \mathrm{S}$ e $60^{\circ} \mathrm{N}$, imageando em 11 dias $80 \%$ da superfície terrestre. O projeto usou grades 
de 90m (SRTM 3) no processamento das imagens e na elaboração de modelos tridimensionais da América do Sul, disponibilizados gratuitamente (CHIEN, 2000).

As imagens SRTM, oriundas do projeto TOPOPDATA (INPE, 2009) são disponibilizadas gratuitamente no site do INPE na escala de 1:250.000, projetadas em Datum WGS84 e organizadas em quadrículas de $1^{\circ}$ de latitude por $1^{\circ} 30^{\prime}$ de longitude, formando uma grade conhecida como GRADE TOPODATA. Para realizar o presente estudo foram necessárias quatro imagens TOPODATA para abranger toda a área das bacias de contribuição dos reservatórios Umari e Mendubim (Figura 2).

O pré-processamento e processamento das TOPODATAS foi realizado utilizando o programa livre QGIS 2.18, disponibilizado no site da Comunidade QGISBRASIL. Como produto do pré-processamento, foi gerado um mosaico, reprojetado para o Datum SIRGAS 2000 UTM zona 24S, com as informações SRTM das imagens originais, para cada uma das áreas de interesse. Para a extração dos limites das bacias em estudo, definidos pelos seus divisores de água, e de suas redes de drenagem foi utilizada a ferramenta TauDEM do programa QGis. Como produtos do processamento dessas imagens, foram gerados os "Shapes" poligonais e de linhas, respectivamente, dos "layouts" e da rede de drenagem dessas bacias.

Figura 2: Recorte da grade TOPO DATA, com destaque para as imagens utilizadas na morfometria das áreas em estudo.

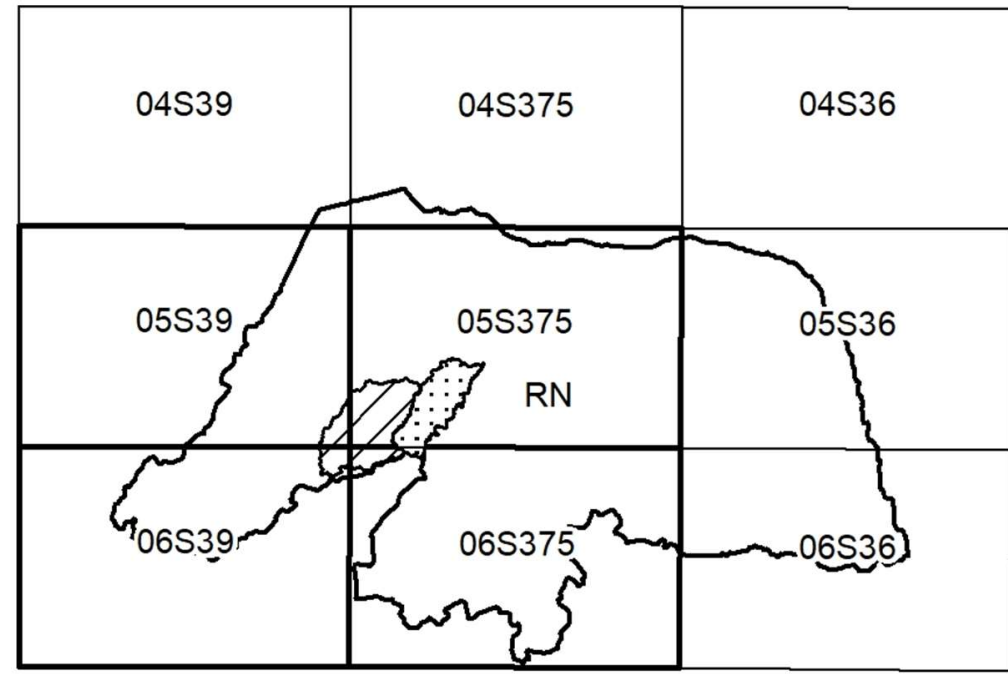

Convenções

53 Bacia de contribuição do Reservatório Umari

¿3 Bacia de contribuição do Reservatório Mendubim

Grade TOPODATA

Fonte: Autores (2019). 


\section{Parâmetros morfométricos}

A análise das bacias foi dividida em dois níveis: hipsométrica e morfométrica (análise linear e areal). Os parâmetros morfométricos, descritos a seguir, foram calculados de acordo com Antoneli; Thomaz (2007); Andrade et al. (2008); Coutinho et al. (2011) (Anexo I).

\section{Hipsometria}

A altitude mínima refere-se a cota do ponto exutório da bacia, e a altitude máxima refere-se a cota do ponto extremo montante do eixo da bacia, sendo a diferença entre estas cotas a amplitude altimétrica da bacia de contribuição dos reservatórios. $\mathrm{O}$ gradiente do canal principal (G) expresso em percentagem, esse índice indica a declividade do canal principal, relacionando sua cota máxima com seu comprimento (HORTON, 1945). A relação de relevo (Rr) é a relação entre a amplitude de altimétricas (considerando como cota mínima a foz do canal principal e como cota máxima, a maior cota oposta a foz) e o comprimento da linha mais longa da bacia paralela à drenagem principal (canal principal), baixos valores de Rr, indicam baixa declividade (SCHUMN, 1956). O índice de rugosidade (Ir) expressa o potencial erosivo de uma bacia em função do escoamento superficial, ou seja, quanto menor o valor de Ir, menor será a degradação a que a bacia está exposta (TRAJANO et al., 2012). O índice de sinuosidade (Is) avalia o layout dos canais, valores muito próximos da unidade indicam canais retilíneos; valores intermediários (entre 1 e 2) indicam canais de forma transitória, entre sinuosos e retilíneos; e valores acima de 2 indicam canais sinuosos (ALVES; CASTRO, 2003).

\section{Morfometria}

Análise linear

Na hierarquização de Strahler (1954), são atribuídos níveis de ordem aos canais. Os canais que correspondem às nascentes são denominados de primeira ordem, os canais que recebem a contribuição desses são denominados de segunda ordem, os que recebem desses, de terceira ordem, assim por diante. Um canal só é promovido à ordem posterior, quando recebe contribuição hídrica de, pelo menos, dois canais da ordem anterior. Ao final da classificação, a ordem da bacia é definida pela maior ordem de seus canais. A relação ponderada de bifurcação $(\mathrm{Rpb})$ tem como objetivo calcular um valor de bifurcação que seja mais representativo para a bacia como um todo. Os valores da Rpb variam entre 3,0 e 5,0 (PANDE; MOHARIR, 2017). Nos sistemas de drenagem 
classificados pelo modelo de hierarquização de Strahler (1954), o valor da relação de bifurcação (Rb) deverá ser maior ou igual 2,0 (CHRISTOFOLETTI, 1980).

\section{Análise areal}

O fator de forma (kf) é definido como a razão entre a área de uma bacia e o quadrado de seu comprimento, sendo sempre maior que 0,78 para bacias perfeitamente circulares. Para bacias longas e estreitas este fator pode estar ligado ao regime de inundação, no entanto, para bacias irregulares este fator não é sensível às caraterísticas hidrológicas, principalmente em bacias de solo permeável (HORTON, 1932).

O índice de compacidade $(\mathrm{Kc})$ ou coeficiente de compacidade, relaciona o perímetro de uma bacia com a circunferência de um círculo de área igual a da bacia (CARDOSO et al., 2006). Quanto mais próximo da unidade o valor de Kc, mais susceptível é uma bacia a enchentes acentuadas. Já o índice de circularidade (IC) relaciona a área de uma bacia com seu perímetro; este parâmetro é importante para avaliar a susceptibilidade de uma bacia a cheias rápidas, em funções de um maior ou menor escoamento. Um $\mathrm{IC}=0,51$ indica uma bacia de escoamento moderado, e com pequena propensão a cheias rápidas; IC $>0,51$ bacia com forte tendência circular, muito susceptível a enchentes; $\mathrm{IC}<0,51$ bacias alongadas, favorecimento do escoamento e menor probabilidade de cheias rápidas (ALVES; CASTRO, 2003).

Os valores de densidade de drenagem (Dd) avaliam o escoamento de uma bacia hidrográfica e o grau de infiltração, além de sua tendência à erosão. Valores menores que 0,5 são considerados baixos; entre 0,5 e 2,0 medianos e entre 2,01 e 3,5 altos, e maiores que 3,5 muito altos (DA VEIGA BELTRAME, 1994), enquanto que o coeficiente de manutenção $(\mathrm{Cm})$ define menor valor de área $\left(\mathrm{km}^{2}\right)$ necessária para manter um quilometro de canal de escoamento. Esse parâmetro corresponde ao recíproco da densidade de drenagem (SCHUMN, 1956).

A densidade hidrográfica avalia a capacidade que tem a bacia de formar novos cursos d'água em função das características edafoclimáticas da região (CASTRO; CARVALHO, 2009), além de avaliar também o potencial hídrico da bacia em função do número de canais por $\mathrm{km}^{2}$; quanto menor o valor desse parâmetro, menor o potencial hídrico da bacia (ALVES; CASTRO, 2003). Já a extensão do percurso superficial (Eps), indica a distância média que uma enxurrada percorre do interflúvio ao canal permanente, sendo um fator importante para avaliar a hidrologia de uma bacia em função da sua fisiografia (BARBOSA; CARVALHO, 2009). 


\section{RESULTADOS E DISCUSSÕES}

\section{Análise hipsométrica}

Os parâmetros hipsométricos encontram-se na tabela 1 e os mapas temáticos de distribuição das classes altimétricas encontradas nas bacias de contribuição dos reservatórios de Umari e Mendubim estão na figura 3.

Tabela 1 - Parâmetros hipsométricos das bacias de contribuição dos reservatórios Umari e Mendubim.

\begin{tabular}{lcrr}
\hline \multirow{2}{*}{ PARÂMETROS } & \multirow{2}{*}{ Unid. } & \multicolumn{2}{c}{ Reservatório } \\
\cline { 3 - 4 } & & Mendubim & Umari \\
\hline Altitude máxima (Hmáx.) & $\mathrm{m}$ & 575,00 & 695,00 \\
Altitude mínima (Hmín.) & $\mathrm{m}$ & 25,00 & 45,00 \\
Amplitude altimétrica (Hm) & $\mathrm{m}$ & 550,00 & 651,00 \\
Gradiente canal principal (G) & $\%$ & 0,95 & 2,08 \\
Relação de relevo (Rr) & $\mathrm{m} / \mathrm{km}$ & 9,04 & 10,07 \\
Índice de rugosidade (Ir) & $\mathrm{Adm}$. & 0,27 & 0,48 \\
Índice de Sinuosidade (Is) & Adm. & 1,15 & 1,23 \\
\hline
\end{tabular}

As bacias de Umari e Mendubim apresentaram elevadas amplitudes altimétricas, sendo que a maior parte dessas bacias abrangem áreas com altitudes entre 50 e $250 \mathrm{~m}$, ou seja, $83,8 \%$ da área de Umari e $77 \%$ da área de Mendubim. Analisando os gradientes dos canais principais de ambas as bacias, se observa um decaimento suave e baixa declividade, contribuindo para uma velocidade de escoamento reduzida, que tende a favorecer a redução das taxas de erosão.

Quando analisamos os perfis topográficos longitudinais dos eixos das bacias, verificamos que a bacia de Umari, em sua porção mais a montante (entre 60 a $65 \mathrm{~km}$ do ponto exutório), apresenta um setor de maior fragilidade, com um possível aumento dos processos erosivos (Figura 4). Na bacia de contribuição do reservatório de Mendubim a variação de altitude, ao longo de seu perfil longitudinal, é menos abrupta que a de Umari. O relevo da bacia de contribuição de Mendubim é mais acidentado, favorecendo o acúmulo de material oriundo da erosão e também o aumento da sua velocidade de escoamento em relação à de Umari. 
Figura 3 - Altimetria da bacia hidrográficas de contribuição do reservatório Umari
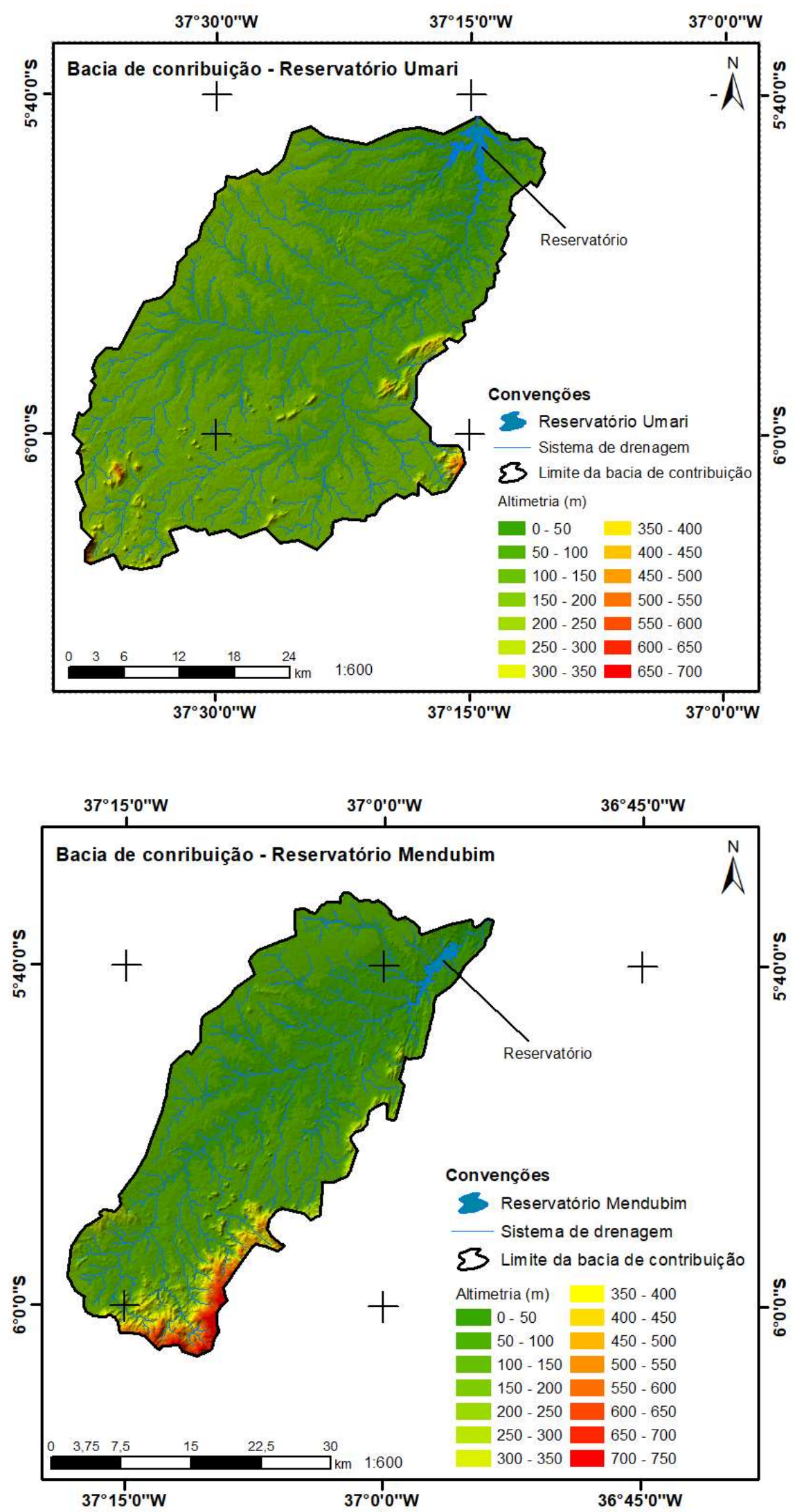

Fonte: Autores, 2019. 
Figura 4 - Perfis longitudinais das bacias hidrográficas dos reservatórios Umari e Mendubim.

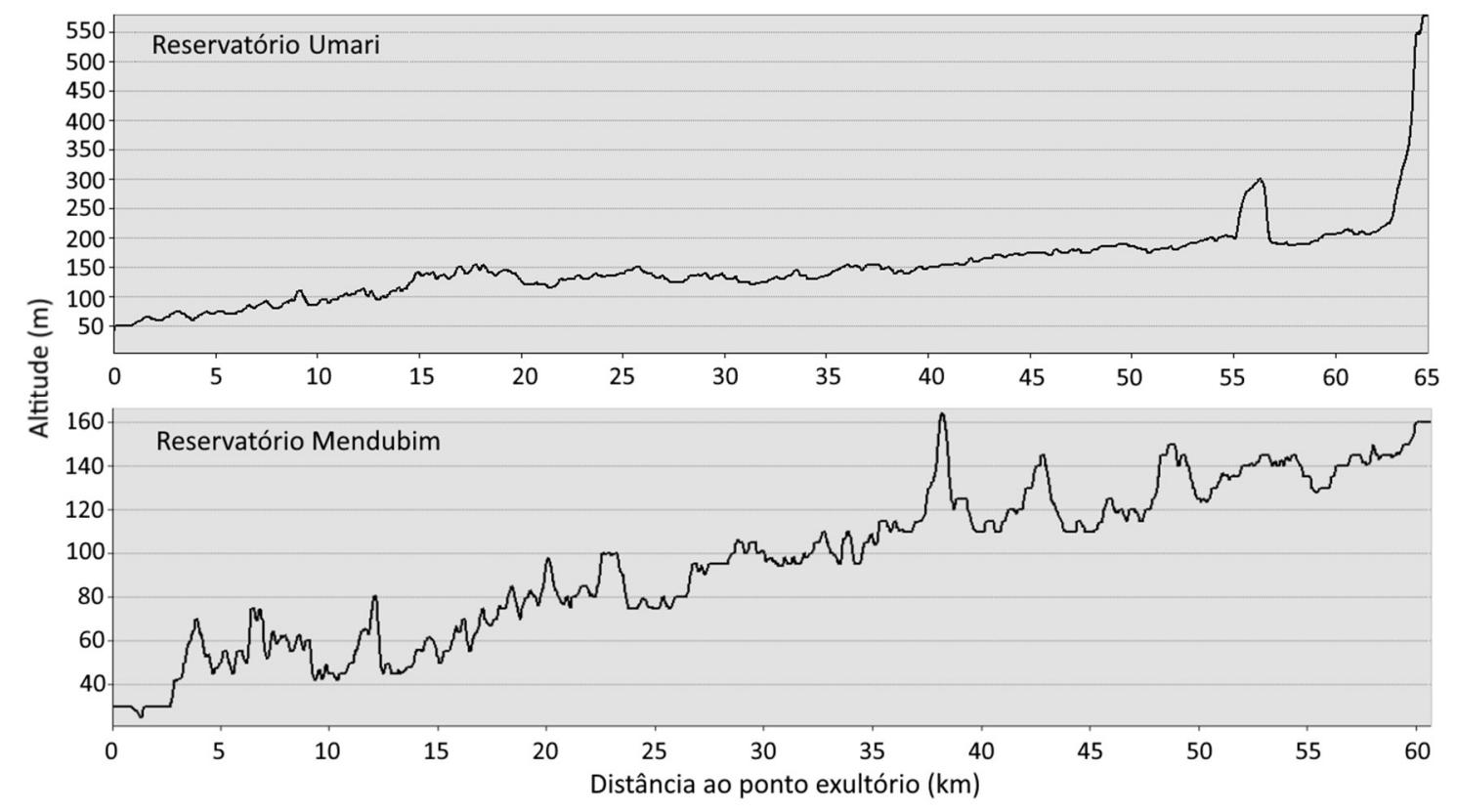

Fonte: Autores, 2019

A maior velocidade de escoamento torna a bacia de Mendubim menos susceptível a enchentes e mais susceptível a erosão, quando comparada a de Umari, visto que o aumento dessa velocidade acentua o processo de erosão (SANTOS, 2005). O índice de rugosidade da bacia de Umari $(\mathrm{Ir}=0,48)$ indica que esta é mais sujeita a degradação por processos erosivos, que a bacia de Mendubim ( $\operatorname{Ir}=0,27)$. As bacias de Umari e Mendubim apresentaram valores baixos e muito aproximados para a relação de relevo $(\mathrm{Rr})$, respectivamente, 9,04 e 10,07, indicando que ambas possuem baixas declividade e velocidades de escoamento.

De acordo com Schumm (1963), o formato dos canais superficiais está relacionado com a velocidade de escoamento e com o acúmulo de sedimentos. Sendo assim, é de suma importância avaliar a morfometria do canal principal desse tipo de bacia, para entender melhor a hidrodinâmica superficial. Os canais principais das bacias de contribuição dos reservatórios de Umari e Mendubim apresentaram índices de sinuosidades intermediários ( $\mathrm{Is}=1,35$ e 1,34, respectivamente), levando a supor que há um relativo equilíbrio entre o transporte e acúmulo de sedimentos. 
Análise morfométrica

A análise morfométrica foi dividida em análise linear e análise areal. Esta análise permitiu avaliar a estrutura das bacias em função dos índices e parâmetros morfoestruturais de sua rede de drenagem (Tabela 2).

Tabela 2: Parâmetros morfométricos das bacias de contribuição dos reservatórios Umari e Mendubim. Adm.: Adimensional.

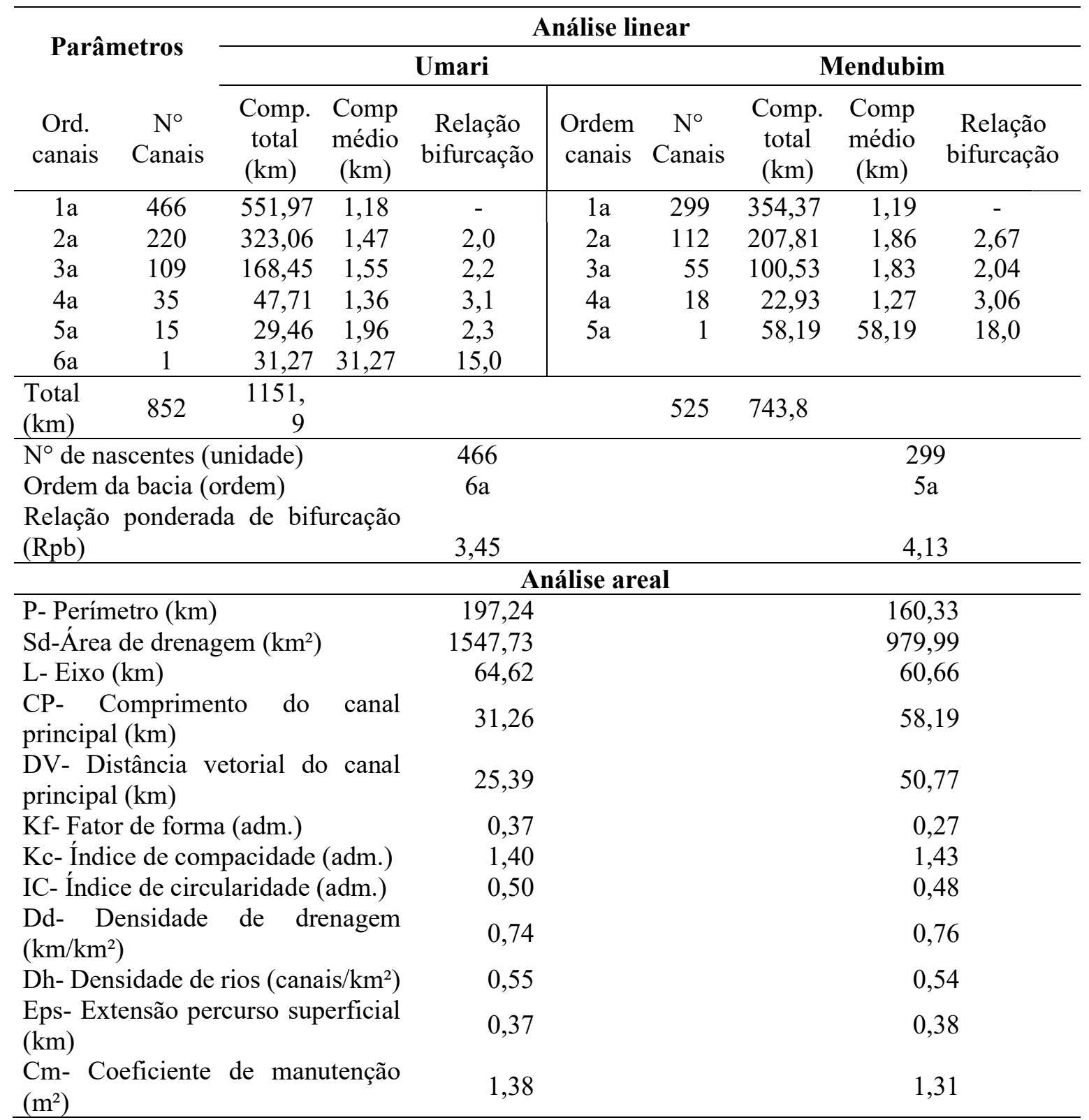

De acordo com a classificação pelo método de Strahler (1954), a bacia do reservatório Umari foi classificada como de sexta ordem e a do Mendubim como de quinta (Figura 5, Tabela 2). 
Figura 5 - Hierarquia das redes de drenagem das bacias de contribuição dos reservatórios Umari e Mendubim, de acordo com Strahler (1954).
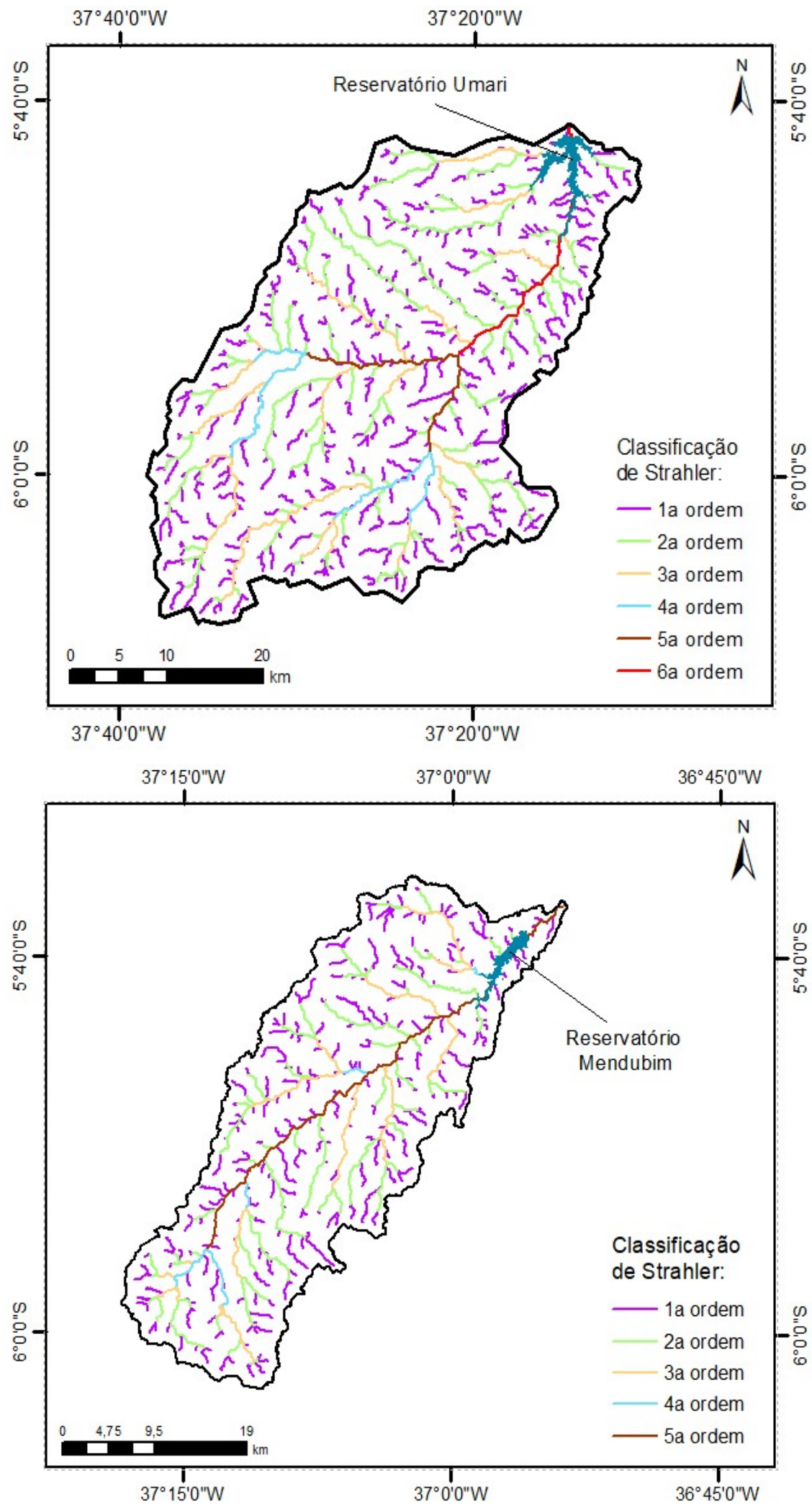

Fonte: Autores, 2019. 
Valores altos de Rpb indicam menor escoamento superficial e uma maior permeabilidade dos substratos das bacias (BARBOSA; CARVALHO, 2009). A bacia de contribuição do reservatório Umari apresentou $\mathrm{Rpb}(4,13)$ maior que a de Mendubim $(3,45)$, isso indica que a de Mendubim tende a ter um escoamento superficial maior com solos menos permeáveis. As bacias de contribuição dos reservatórios apresentaram índices de circularidade semelhantes, Umari $(\mathrm{IC}=0,50)$ e Mendubim $(\mathrm{IC}=0,47)$, esses valores são característicos de bacias alongadas, que favorecem o escoamento, reduzindo a probabilidade de cheias rápidas. $\mathrm{O}$ que vem a ser confirmado pelo índice de compacidade $(\mathrm{Kc})$. Bacias que apresentam $\mathrm{Kc}$ entre 1,25 e 1,50, são consideradas com tendência mediana a enchentes acentuadas (GEORGIN; OLIVEIRA; ROSA, 2015). Para esse parâmetro, as bacias de contribuição também apresentaram valores semelhantes, Umari $(\mathrm{Kc}=1,40)$ e Mendubim $(\mathrm{Kc}=1,43)$, ratificando que essas bacias possuem tendência mediana a cheias rápidas. No entanto, os valores do fator de forma das bacias de contribuição de Umari $(\mathrm{Kf}=0,37)$ e Mendubim $(\mathrm{Kf}=0,27)$ demonstraram que, comparativamente, a primeira tende a ser mais susceptível a enchentes, visto que quanto menor esse fator, menor é a probabilidade de enchentes (ANDRADE et al., 2008; GEORGIN; OLIVEIRA; ROSA, 2015).

Os valores de densidade de drenagem das bacias de contribuição dos reservatórios, Umari $(\mathrm{Dd}=0,74)$ e Mendubim $(\mathrm{Dd}=0,76)$, foram semelhantes e medianos, demonstrando um relativo equilíbrio entre escoamento e infiltração. Esse parâmetro varia com as características edafoclimáticas de uma região, e sua ação sobre o comportamento hidrológico das rochas, em terrenos cujo substrato rochoso permite uma boa infiltração, o escoamento superficial é minimizado, prejudicando a formação de canais, reduzindo os valores da Dd (CHRISTOFOLETTI, 1980). O que vem a ser reafirmado pelos baixos valores da densidade hidrográfica de ambas as bacias (0,55 para Umari e 0,54 para Mendubim).

As bacias analisadas apresentaram baixos potenciais hídricos e de contribuição para os reservatórios Umari e Mendubim. Este fato reflete no elevado valor do coeficiente de manutenção $(\mathrm{Cm})$ apresentado por ambas. Como as bacias de contribuição apresentaram baixo potencial hídrico, isso elevou os valores do Cm (Umari - 1,38 km²) e (Mendubim -1,31 km²). As bacias de contribuição apresentaram ainda valores reduzidos de Extensão de Percurso Superficial (Umari - 0,37 km e Mendubim $0,38 \mathrm{~km}$ ), demonstrando um escoamento rápido das águas pluviais até seus respectivos rios principais. 


\section{CONSIDERAÇÕES FINAIS}

As bacias de contribuição dos reservatórios Umari e de Mendubim apresentaram características morfométricas semelhantes. São bacias de extensões medianas, com formato alongado, pouca tendência a enchentes, sendo a bacia de contribuição do reservatório Umari mais susceptível do que a do reservatório Mendubim. Apesar das elevadas amplitudes altimétricas, seus canais principais apresentam baixa declividade e velocidade de escoamento. Alguns dos parâmetros analisados permitem algumas inferências a respeito da permeabilidade dos substratos de bacias hidrográficas, considerando esses parâmetros, supõe-se que os substratos das bacias de contribuição estudadas tendem ser permeáveis, mas com um equilíbrio relativo entre infiltração e escoamento, sendo que a bacia de contribuição do reservatório Mendubim tende a ter um substrato mais permeável que a de Umari. As redes de drenagem dessas bacias apresentaram grau de dissecação mediano. Devido às baixas densidades de drenagem e hidrográfica, essas bacias se mostraram com baixo potencial de contribuição hídrica superficial para seus principais reservatórios. Por fim, concluímos que a aplicação de técnicas de geoprocessamento em modelos digitais de elevação (MDE) e a ferramenta TauDem ("Terrain Analysis Using Digital Elevation Models"), do módulo de pesquisa hidrológicas do programa QGis 2.18, se mostraram eficientes na extração dos dados necessários às análises, hipsométrica e morfométrica, bem como para a caracterização das bacias de contribuição estudadas.

\section{REFERÊNCIAS BIBLIOGRÁFICAS}

AHER, P. D.; ADINARAYANA, J.; GORANTIWAR, S. D. Quantification of morphometric characterization and prioritization for management planning in semi-arid tropics of India: A remote sensing and GIS approach. Journal of Hydrology, v. 511, p. 850-860, 2014. http://dx.doi.org/10.1016/j.jhydrol.2014.02.028.

ALVES, J. M. D. P.; CASTRO, P. D. T. A. Influência de feições geológicas na morfologia da Bacia do Rio Tanque (MG) baseada no estudo de parâmetros morfométricos e análise de padrões de lineamento. Revista Brasileira de Geociências, v. 33, n. 2, p. 117-224, junho 2003.

ANDRADE, N. L. R. et al. Caracterização Morfométrica e Pluviométrica da Bacia do Rio Manso-MT. Geociência, São Paulo, v. 27, n. 2, p. 237-248, 2008.

BAPTISTA, M. N. et al. Selection of Preferred Floodplains for the Renaturalization of Hydrologic Functions: A Case Study of the Paraíba do Sul River Basin, Brazil. Water resources management, v. 28, n. 13, p. 4781-4793, 2014. 
BARBOSA, Y. B.; CARVALHO, S. M. Análise morfométrica da bacia do Arroio do Padre, Ponta Grossa - PR. CAMINHOS DE GEOGRAFIA - revista on line, v. 10, $\mathrm{n}$. 30, p. 160-173, Mar 2009. ISSN ISSN 1678-6343. Disponível: http://www.ig.ufu.br/revista/caminhos.htm.

BENTES-GAMA, M. Manejo de bacias hidrográficas. [S.1.]: Artigos técnico, EMBRAPA- Empresa Brasileira de Pesquisa Agropecuárias, v. 14, 2003.

BRASIL. Lei ${ }^{\circ} 9.433$ de 08 de janeiro de 1997. Institui a Política Nacional dos Recursos Hídricos, cria o Sistema Nacional de Gerenciamento de Recursos Hídricos, Brasíla, 08 jan 1997.

CARDOSO, C. A. et al. Caracterização morfométrica da bacia hidrográfica do Rio Debossan, Nova Friburgo, RJ. Revista Árvore, p. 214-248, 2006. ISSN 0100-6762.

CASTRO, S. B.; CARVALHO, T. M. Análise morfométrica e geomorfologia da bacia hidrográfica do rio Turvo-GO, através de técnica de sensoriamento remoto $\mathrm{e}$ geoprocessamento. SCENTIA PLENA, v. 5, n. 2, 2009.

CHIEN, P. Endeavour maps the world in three dimensions. Geoword, v. 37, p. 32-38, 2000 .

CHRISTOFOLETTI, A. Geomorfologia. 2. ed. São Paulo: Edgard Blucher, 1980. 188 p.

CUNHA, E. R. D.; BACANI, V. M.; PANACHUKI, E. I. Modeling soil erosion using RUSLE and GIS. Natural Hazards, v. 85, n. 2, p. 851-868, 2016.

DA VEIGA BELTRAME, A. Diagnóstico do meio físico de bacias hidrográficas: modelo e aplicação. Florianópolis: UFSC, 1994.

GEORGIN, J.; OLIVEIRA, G. A.; ROSA, A. L. D. D. Estudo comparativo de índices morfométricos relacionado com cheias nas bacias hidrográficas do alto Jacuí e Vacacaí Vacacaí Mirim -RS. Revista Eletrônica em Gestão, Educação e Tecnologia Ambiental, Santa Maria, v. 19, n. 2, p. 1357-1364, mai-ago 2015. ISSN ISSN : 22361170. Acesso:https://periodicos.ufsm.br/reget/article/viewFile/16834/pdf.

HORTON, R. E. Drainage-basin characteristics. Eos, Transactions American Geophysical Union, 1932.

INPE. TOPODATA - Banco de Dados Geomorfomértricos do Brasil, 2009. Disponivel em: <http://www.dsr.inpe.br/topodata/acesso.php>. Acesso em: 14 jan 2016.

IWASHITA, F.; FRIEDEL, M. J.; FERREIRA, F. J. F. A self-organizing map approach to characterizehydrogeology of the fractured Serra-Geral transboundaryaquifer. Hydrology Research, v. 49, n. 3, p. 794-814, 2017.

KÖPPEN, W.; GEIGER, R. Classificação de Köppen. [S.1.]: [s.n.], 1936.

MEDEIROS, F. S. D. et al. Analysis of Morphometric Variables of River Espinharas Hydrographic Sub-basin Using Geographic Information System. Journal of Experimental Agriculture International, v. 31, n. 6, p. 1-18, 2019. https://doi.org/10.9734/jeai/2019/v31i630088. 
MENEZES, J. P. C. D. et al. Morfometria e evolução do uso do solo e da vazão de máxima em uma micro bacia urbana. Revista Brasileira de Geomorfologia, v. 15, n. 4, p. 659672, 2014. ISSN 2236-5664.

OLIVEIRA, M. A.; BARBOSA, E. N.; DANTAS NETO, J. Gestão de recursos hídricos no Rio Grande do Norte: una análise da implementação da política hídrica. HOLOS, p. 3-25, 2013. ISSN ISSN 1518-1634.

PANDE, C. B.; MOHARIR, K. GIS based quantitative morphometric analysis and its. Applied Water Science, v. 7, n. 2, p. 861-871, 2017.

RÊGO, M. D. F. D. F.; TRAJANO, D. M. D. A. Proposta de Instituição do Comitê da Bacia Hidrográfica do Rio Piranhas-Açu, conforme Resolução $n^{\circ}$ 5, de 10 de abril de 2000, do Conselho Nacional de Recursos Hídricos. ANA - Agência Nacional de Águas. [S.1.]. 2005.

SANTOS, E. D. Mapeamento da fragilidade ambiental da bacia hidrográfica do rio Jirau, minicípio de Dois Vizinhos-Paraná, 2005.

SCHUMM, S. A. Evolution of drainage systems and slopes in badlands at perth amboy, New Jersey. The Geological Society of America: GSA Bulletin, v. 67, n. 5, p. 571-596, May 1956. ISSN doi:10.113.

SILVA, M. T. et al. Morfometric analysis of the basin low middle São Francisco river. Journal of Hyperspectral Remote Sensing, v. 4, n. 8, p. 168-174, 2014. ISSN 22372202.

SOUZA, A. C. M.; SILVA, M. R. F.; DIAS, N. S. Gestão de Recursos Hídricos: O caso da bacia hidrográfica Apodi-Mossoró. Irriga, Botucatu/SP, n. Edição Especial, p. 280296, 2012.

STRAHLER, A. N. Quantitative geomorphology of erosional landscapes. In: CR 19th International Geological Congress, Algiers, 1952. 1954. p. 341-354.

TRAJANO, S. R. R. D. S. et al. Análise Morfométrica de Bacia Hidrográfica: Subsídio à Gestão Territorial Estudo de Caso no Alto e Médio Mamanguape. Embrapa Gestão Territorial-Boletim de Pesquisa e Desenvolvimento (INFOTECA-E), Campinas-SP, 2012.

YUSUF, S. M. et al. The Utilization of Satellite Imagery Data to Predict Hydrology Characteristics in Dodokan Watershed. Procedia Environmental Sciences, p. 36-43, 2016. https://doi.org/10.1016/j.proenv.2016.03.054. 
Anexo I - Equações de cálculo dos parâmetros morfométricos

\begin{tabular}{|c|c|c|c|}
\hline Parâmetro & Equação & Und. & Elementos \\
\hline Relação de relevo (Rr) & $\mathrm{Rr}=\frac{\mathrm{Hm}}{\mathrm{L}}$ & $\mathrm{m} / \mathrm{km}$ & $\begin{array}{l}\text { Hm = Cota máxima }- \text { Cota min } \\
\text { (amplitude altimétrica }-\mathrm{m}) \text {; } \\
\text { L: comprimento do canal } \\
\text { principal }(\mathrm{km}) \\
\text { Obs.: A cota mínima } \\
\text { corresponde à foz do canal } \\
\text { principal; e a máxima a maior } \\
\text { cota encontrada em um ponto } \\
\text { da bacia, oposto a foz. }\end{array}$ \\
\hline fado canal principal $(\mathrm{G})$ & $G=\frac{\text { Hmáx. }}{10 x \mathrm{~L}}$ & $\%$ & $\begin{array}{l}\text { H máx.: Cota máxima da bacia } \\
\text { (m) } \\
\text { L: comprimento do canal } \\
\text { principal }(\mathrm{km}) \\
\text { Obs.: valor já convertido para } \\
\text { percentual. }\end{array}$ \\
\hline $\begin{array}{l}\text { Índice de Sinuosidade } \\
\text { (Is) }\end{array}$ & $I s=\frac{L}{D v}$ & Adm. & $\begin{array}{l}\text { L: comprimento do canal } \\
\text { principal }(\mathrm{km}) \\
\text { Dv: Distância vetorial (linha } \\
\text { reta entre os extremos do canal } \\
\text { principal) }\end{array}$ \\
\hline Índice de rugosidade (Ir) & $I r=\frac{D d x H m}{1000}$ & Adm. & $\begin{array}{l}\text { Dd: Densidade de drenagem } \\
\left(\mathrm{km} / \mathrm{km}^{2}\right) \\
\text { Hm.: Amplitude altimétrica da } \\
\text { bacia }(\mathrm{m}) \\
\text { Divisão por } \\
\text { transformação da Hm de metros } \\
\text { par quilômetros. }\end{array}$ \\
\hline $\begin{array}{l}\text { Relação ponderada de } \\
\text { bifurcação (Rpb) }\end{array}$ & $R b p=\frac{\sum_{1}^{n} R b_{n}\left(c_{n}+c_{n+1}\right)}{\sum C}$ & Adm. & $\begin{array}{l}\text { Rb: Relação de bifurcação de } \\
\text { cada ordem da classificação de } \\
\text { Strahler; } \\
\text { C: } N^{\circ} \text { de canais de cada ordem; } \\
\text { n: ordem na classificação de } \\
\text { Strahler. }\end{array}$ \\
\hline $\begin{array}{l}\text { Relação de bifurcação } \\
(\mathrm{Rb})\end{array}$ & $\mathrm{Rb}=\frac{\mathrm{N}_{\mathrm{x}}}{\mathrm{N}_{\mathrm{x}+1}}$ & Adm. & $\begin{array}{l}\mathrm{N}_{\mathrm{x}} \text { : Número de seguimentos de } \\
\text { uma ordem; } \\
\mathrm{N}_{\mathrm{x}+1} \text { : Número de seguimentos } \\
\text { de uma ordem imediatamente } \\
\text { superior; }\end{array}$ \\
\hline Fator de forma $(\mathrm{kf})$ & $\mathrm{kf}=\frac{\mathrm{A}}{\mathrm{L}^{2}}$ & Adm. & $\begin{array}{l}\text { A: Área da bacia }\left(\mathrm{km}^{2}\right) \\
\mathrm{L}: \text { comprimento do canal } \\
\text { principal }(\mathrm{km})\end{array}$ \\
\hline $\begin{array}{l}\text { Índice de compacidade } \\
(\mathrm{kc})\end{array}$ & $\mathrm{kc}=0,28 \frac{\mathrm{P}}{\sqrt{A}}$ & Adm. & $\begin{array}{l}\text { P: Perímetro da bacia }(\mathrm{km}) \\
\text { A: Área da bacia }\left(\mathrm{km}^{2}\right)\end{array}$ \\
\hline $\begin{array}{l}\text { Índice de circularidade } \\
\text { (IC) }\end{array}$ & $\mathrm{IC}=\frac{12,57 x A}{\mathrm{P}^{2}}$ & Adm. & $\begin{array}{l}\text { A: Área da bacia }\left(\mathrm{km}^{2}\right) \\
\text { P: Perímetro da bacia }(\mathrm{km})\end{array}$ \\
\hline
\end{tabular}




\begin{tabular}{l|c|c|l}
\hline Parâmetro & Equação & Und. & \multicolumn{1}{|c}{ Elementos } \\
\hline $\begin{array}{l}\text { Densidade de drenagem } \\
(\mathrm{Dd})\end{array}$ & $D d=\frac{L t}{A}$ & $\begin{array}{c}\mathrm{km} / \mathrm{k} \\
\mathrm{m}^{2}\end{array}$ & $\begin{array}{l}\mathrm{Lt}=\text { comprimento total dos } \\
\text { canais }(\mathrm{km}) \\
\mathrm{A}=\text { Área da bacia }\left(\mathrm{km}^{2}\right)\end{array}$ \\
\hline $\begin{array}{l}\text { Densidade hidrográfica } \\
(\mathrm{Dh})\end{array}$ & $D h=\frac{N}{A}$ & $\begin{array}{l}\text { canais } \\
/ \mathrm{km}^{2}\end{array}$ & $\begin{array}{l}\mathrm{Dh}=\text { Densidade hidrográfica } \\
\mathrm{N}=\text { Número de rios ou canais } \\
\mathrm{A}=\text { Área da bacia }\left(\mathrm{km}^{2}\right)\end{array}$ \\
\hline $\begin{array}{l}\text { Extensão do percurso } \\
\text { superficial (Eps) }\end{array}$ & Eps $=\frac{1}{2 D d}$ & $\mathrm{~km}$ & $\mathrm{Dd}=$ Densidade de drenagem \\
\hline $\begin{array}{l}\text { Coeficiente } \\
\text { manutenção (Cm) }\end{array}$ & $C m=\frac{1}{D d}$ & $\mathrm{~m}^{2}$ & $\mathrm{Dd}=$ Densidade de drenagem \\
\hline
\end{tabular}

